[When citing this chapter, refer to Behaviour 152 (2015) 375-406]

\title{
Context influences spatial frames of reference in bonobos (Pan paniscus)
}

\author{
Alexandra G. Rosati * \\ Department of Psychology, Yale University, New Haven, CT, USA \\ *Author's e-mail address: alexandra.rosati@yale.edu
}

Accepted 15 February 2014; published online 14 March 2014

\begin{abstract}
Primates must solve complex spatial problems when foraging, such as finding patchy resources and navigating between different locations. However, the nature of the cognitive representations supporting these types of behaviors is currently unclear. In humans, there has been great debate concerning the relative importance of egocentric representations (which are viewer-dependent) versus allocentric representations (which are based on aspects of the external environment). Comparative studies of nonhuman apes can illuminate which aspects of human spatial cognition are shared with other primates, versus which aspects are unique to our lineage. The current studies therefore examined spatial cognitive development in one of our closest living relatives, bonobos (Pan paniscus) across contexts. The first study assessed how younger bonobos encode locations in a place-response task in which apes first learn that one of two locations is consistently baited with a reward, and then must approach the two locations from a flipped perspective. The second study examined how a larger age sample of bonobos responded to a spatial relations task in which they first experience that one location is baited, and then can generalize this learning to a new set of targets. Results indicated that while bonobos exhibited a predominantly allocentric strategy in the first study, they consistently exhibited an egocentric strategy in the second. Together, these results show that bonobos can use both strategies to encode spatial information, and illuminate the complementary contributions to cognition made by egocentric and allocentric representations.
\end{abstract}

\section{Keywords}

spatial cognition, memory, spatial relations, habit formation, allocentric, egocentric.

\section{Introduction}

Primates in the wild face complex spatial problems. For example, all primates must locate important items in their environment - such as patchilydistributed food resources or other conspecifics - as well as travel efficiently in their environment (Gallistel, 1990; Shettleworth, 1998; Janson \& Byrne, 
2007). Studies of primate foraging both in the wild (Janson, 1998, 2007; Normand \& Boesch, 2009; Normand et al., 2009) and in captivity (Menzel, 1973; Menzel et al., 2002; Mendes, 2008; Rosati \& Hare, 2012) suggest that primates exhibit sophisticated behaviors when solving these types of spatial problems. However, the particular cognitive capacities underlying these behaviors are currently unclear. There are two general strategies that organisms could use for goal-oriented navigation (Maguire et al., 1998; Burgess, 2008). First, an individual could move through the environment using an allocentric strategy that depends on representations of the world at large, by encoding the set of relationships between environmental cues and the relevant goal (such as a food patch). This type of enduring representation of the layout of objects in the environment has been referred to as a 'cognitive map' (Tolman, 1948). Alternatively, an individual could use an egocentric strategy that depends on representations of the organism's movements centered on their own body (e.g., turn left), sometimes called 'dead reckoning'. The relative importance of these types of strategies in human spatial cognition is a topic of debate. Moreover, some hypotheses suggest that language allows humans to use spatial information with a degree of flexibility and accuracy not seen in other species (Hermer-Vazquez et al., 2001; Wang \& Spelke, 2002). Consequently, studies of nonhuman ape cognition can identify the capacities underlying their natural spatial behaviors, as well as illuminate what aspects of human spatial cognition are unique to our lineage.

How might language influence the manner in which humans encode and remember spatial information? First, some research suggests that language influences the coordinate systems that humans use when thinking about space. Whereas some languages encode spatial relations in terms of a viewerrelative system (e.g., the table is to the left or right), others use a system that encodes the absolute position of objects (e.g., the table is to the North or South). The dominant way a given language describes spatial relations is thought to influence the way mature language speakers encode spatial relations in nonverbal spatial relations tasks: children who grow up speaking language with a preponderance of relative constructions - such as English and other European languages - tend to show an egocentric biases in spatial tasks, whereas speakers of indigenous languages in Africa and other parts the world that use absolute constructions exhibit allocentric bias (Brown \& Levinson, 1992, 2000; Levinson, 1996; Pederson et al., 1998; Levinson et al., 2002; Majid et al., 2004). For example, in one relevant task children watch an 
experimenter hide a reward under one cup in a spatial array, and then move to a second table with an identical array of cups. Their orientation relative to the array is therefore flipped $180^{\circ}$, and the child is asked to predict which location will have the target in this new setup. Their predictions in this context concord with their language's dominant relation construction. As apes tend to depend primarily on an allocentric strategy in the same spatial relations task (Haun et al., 2006a), one proposal stemming from this work is that allocentric encoding is phylogenetically ancestral, whereas human-specific cultural influences can override these biologically-based biases so that some humans favor egocentric strategies (see also Gentner, 2007).

An alternative proposal suggests that human spatial memory is supported primarily by egocentric representations of location, which are then combined with geometrical information about the surface of the environment (Wang \& Spelke, 2002). According to this proposal, egocentric representations involve recognizing scenes based on viewer-dependent perspectives, and then updating these egocentric representations based on information about one's own movements. While pre- or nonlinguistic organisms possess geometric representations of the surface geometric of the environment, this information does not directly represent goal-relevant locations in the environment. Thus, this proposal suggests that flexible representations of space integrating both landmark and geometric information require spatial language (e.g., HermerVazquez et al., 1999, 2001). Thus, younger children and animals use only geometric information, failing to integrate salient information about landmarks into their search patterns (Cheng, 1986; Hermer \& Spelke, 1994). Indeed, when adults' language systems are taxed by engagement in a second simultaneous task, they perform like young children (Hermer-Vazquez et al., 1999). This hypothesis thus suggests that language allows geometric and feature information to be integrated to form these flexible spatial representations (Spelke et al., 2010).

These proposals both emphasize the importance of language in supporting human-unique forms of spatial reasoning, but differ in which type of strategy humans alone exhibit. However, current research offers a third possibility: organisms might posses multiple memory systems, with egocentric and allocentric representations coexisting in parallel (Sherry \& Schacter, 1987; Burgess, 2006, 2008). That is, this hypothesis suggests that humans and animals may encode both egocentric representations of space as well 
as allocentric representations of the relations between objects and the environment at large, with the particular strategy used depending on the context at hand. For example, neurobiological studies indicate that parallel memory systems can influence behavior, and that these systems are supported by distinct neural circuits in the brain. One illustrative set of studies involves the responses of rats (Rattus norvegicus) to a memory task in which both types of strategies could be used to locate food (Packard, 2009). In the cross maze task, rodents are placed in a cross-shaped maze and initially learn that one side wing is consistently baited with food. This could be encoded using either an allocentric strategy (e.g., 'the food is in that spatial location'), or an egocentric strategy (e.g., 'turn left to find the food'). The animals' strategy is determined by reversing their start position in the maze so they must approach the side wings from a flipped orientation to assess if they search in the previously-rewarded location (allocentric strategy), or if they turn in the same direction even though this now leads to the opposite wing (egocentric strategy). In this task, early learning is supported by place-based memory such that animals initially exhibit allocentric strategies. However, with repetition animals exhibit a shift towards egocentric strategies (Packard, 2009). Moreover, temporary lesions and other neurochemical manipulations can selective inhibit or strengthen these different types of encoding strategies: the hippocampus selectively impacts allocentric encoding, whereas changes to the basal ganglia influence egocentric encoding (Packard, 1999). Together, this evidence indicates that both allocentric encoding of specific locations in space, and egocentric encoding of particular motor responses, exist in parallel (Poldrack et al., 2001; White \& McDonald, 2002; Poldrack \& Packard, 2003).

Developmental work further supports the claim that both types of representations can control behavior depending on context (Newcombe \& Huttenlocher, 2006). For example, when infants initially experience that something interesting (such as a toy) repeatedly appears to one side, but then are flipped to view the same space from the opposite orientation, they tend to show an egocentric bias. However, this shifts to an allocentric bias over the first year of life (Acredolo, 1978, 1979). Studies using search tasks similarly show that egocentric frameworks are available at an earlier age, whereas older children can successfully use both egocentric and allocentric strategies (Newcombe et al., 1998). Importantly, aspects of the external environment can influence children's strategies: even young babies can use allocentric strategies when 
there are very salient landmarks in the external environment (Acredolo \& Evans, 1980). Similarly, although babies tend to exhibit egocentric biases in unfamiliar experimental rooms, they are more likely to use an allocentric strategy in their own homes (Acredolo \& Evans, 1980). Adults exhibit similar patterns, with a greater preference for using an allocentric frame of reference when there are salient landmark cues that situate the array in a larger spatial context (Li \& Gleitman, 2002). Together, these results indicate that different sources of spatial information may be combined in a weighted fashion, with some information more important in specific contexts than others (Ratliff \& Newcombe, 2008).

Overall, these hypotheses concerning human spatial cognition predict that nonlinguistic apes should favor different spatial strategies. The first proposal suggests that apes use primarily allocentric strategies, but this preference can be 'overwritten' by cultural linguistic influences in humans (Majid et al., 2004; Haun et al., 2006a; Gentner, 2007). The second view suggests that nonhumans generally use an egocentric framework to encode space, and that language enables human-unique forms of allocentric representations (Wang \& Spelke, 2002). The final view suggests that nonhuman apes and humans should be both capable of using egocentric and allocentric encoding strategies, although their preference may vary across contexts (Newcombe \& Huttenlocher, 2006). Consequently, evidence from apes can play an important role in evaluating the role of language in how humans represent spatial relations.

What kinds of strategies do apes use when faced with spatial problems? Several lines of evidence suggest that apes can use allocentric frameworks. For example, chimpanzees (Pan troglodytes) can form allocentric 'cognitive maps' of their surrounding: when chimpanzees are shown multiple hiding places for food in a large field, they later will move between those locations using an optimal route to retrieve the food with the least amount of travel distance (Menzel, 1973). Similarly, wild chimpanzee foraging patterns in tropical rainforests suggest that the apes traveled in direct routes to reach food resources (Normand \& Boesch, 2009). In tasks designed to examine spatial strategies in more controlled settings, apes also exhibit allocentric strategies. For example, all four species of great apes exhibited allocentric strategies in the spatial relations task described previously (Haun et al., 2006a). Apes also exhibit allocentric strategies when they initially watch food being hidden in an array, and then can choose after their orientation is flipped (Hoffman \& 
Beran, 2006; Albiach-Serrano et al., 2010). Similarly, when apes' orientation towards the array was the same during both the baiting event and when making a choice (but they move in the interim), apes appear to predominately use spatial cues (e.g., proximity to the edge of the table) rather than egocentric information (left or right locations) when searching for food (Hribar \& Call, 2011; Hribar et al., 2011). Finally, some tasks have examined the extent to which apes use landmark information about the features of items in the environment (such as color or shape), versus purely spatial information about specific locations in the environment. For example, in one task apes initially watched food being hidden under one container in an array where all containers had different features, but then the locations of the containers are switched before the apes could choose. Some evidence suggests that apes tend to show to choose the same location, ignoring that the container's features had changed (Haun et al., 2006b), whereas other studies show that apes exhibit a greater mixture of spatial strategies (Kanngiesser \& Call, 2010). Importantly, however, apes showed a greater tendency to use feature information in tasks involving longer periods of experience (Kanngiesser \& Call, 2010), which might suggest the acquisition of a more habit-based response over time. Nevertheless, current evidence overall suggests that apes have a bias towards allocentric strategy rather than an egocentric strategy.

However, current comparative data on spatial cognition in apes cannot address the relative importance of these strategies for two reasons. First, although humans are equally related to both chimpanzees (P. troglodytes) and bonobos (P. paniscus), chimpanzees are much more commonly studied than are bonobos (Hare, 2007). For example, in the previous studies that explicitly contrasted allocentric versus egocentric frameworks, few bonobos were tested (three to six across studies; Haun et al., 2006a; Albiach-Serrano et al., 2010; Hribar \& Call, 2011). Importantly, differences in the feeding ecology of bonobos and chimpanzees suggest that these species face different spatial challenges while foraging. In particular, chimpanzees are thought to face more patchy, uncertain environments than bonobos (White \& Wrangham, 1988; Kano, 1992; Malenky \& Wrangham, 1993; White, 1998; Hare et al., 2012). This difference in feeding ecology predicts that chimpanzees would show more accurate spatial cognition and memory than bonobos due to their increased reliance of patchily-distributed foods. Cognitive evidence supports 
this prediction: while both chimpanzees and bonobo can successfully recall the location of hidden food in a large space, chimpanzees are more successful at recalling multiple food items than bonobos (Rosati \& Hare, 2012).

Second, there is little data addressing whether apes show any ontogenetic changes in spatial cognition. This is critical for assessing hypotheses pointing to a causal role for language in shaping human spatial cognition: if language drives a particular ontogenetic change in humans, then that developmental shift should be found in our species alone (e.g., Matsuzawa et al., 2006; Matsuzawa, 2007; Rosati \& Hare, 2012; Rosati et al., in press). However, it is currently unclear if nonhuman apes show any human-like shifts in spatial frameworks. For example, the study by Haun and colleagues (2006a) involved only sub-adult and adult apes, and did not examine whether apes exhibited age-dependent change in their preferred strategy. While some studies suggest improvement with age in some memory tasks (Herrmann et al., 2010; Martin-Ordas et al., 2010) these studies did not include infants in the age range where some human changes in spatial skills occur (e.g., Newcombe $\&$ Huttenlocher, 2006). Other studies of infants have been limited to small samples of three or fewer chimpanzees (Matsuzawa et al., 2006; Matsuzawa, 2007). Finally, the few previous studies of spatial cognition involving larger samples sizes of infant and juvenile apes have focused on whether apes could remember particular locations, not the specific strategy they used to do so do (e.g., Rosati \& Hare, 2012; Wobber et al., 2013).

The current paper therefore examines spatial cognition in bonobos in two tasks that have both been commonly used to assess allocentric versus egocentric encoding of locations. The first study examines how bonobos encode locations in the place-response task, which has been used to disentangle allocentric, hippocampal-dependent strategies from egocentric basal-gangliadependent strategies. This study focuses on younger apes $(N=10)$ between the ages of 2 and 7 years. The second study uses a modified version of the spatial relations task previously developed by Haun and colleagues (2006a) to examine linguistic influences on spatial cognition. This study examines a larger sample of bonobos $(N=19)$ ranging in age from 2 to 13 years. Thus, these studies can compare the types of strategies bonobos used in these two different contexts. In addition, these studies can assess whether bonobos exhibit any developmental shift in strategies, suggesting that such shifts might reflect intrinsic maturation of spatial abilities, not the influence of language. 


\section{Study 1: place-response task}

This study adapted the cross-maze task used in studies of rodent (Packard, 2009) and lemur memory (Rosati et al., 2014) for use with infant and juvenile bonobos. Apes were presented with two overturned bowls places on opposite sides of the room, which they could approach to search for food. As in the cross maze task, one of the locations was consistently baited with (hidden) food across sessions. The ape then completed probe trials from a flipped orientation towards the cups to assess if they had encoded the baited option from a spatial (allocentric) perspective or a habitual (egocentric) perspective.

\subsection{Methods}

\subsubsection{Ethics statement}

All behavioral studies were noninvasive. The studies had IACUC approval from Duke University (No. A078-08-03), strictly adhered to the legal requirements of the Democratic Republic of Congo, and had approval from the Ministry of Research and the Ministry of Environment in the DRC (research permit No. MIN.RS/SG/004/2009). Animal husbandry and research practices complied with the PASA Primate Veterinary Healthcare Manual and the policies of Lola ya Bonobo Sanctuary in Kinshasa, Democratic Republic of Congo. All apes were socially housed, and the vast majority semi-free-ranged in large tracts of tropical forest during the day (5-40 ha across groups). In the evening, all apes spent the night in indoor dormitories $\left(12-160 \mathrm{~m}^{2}\right)$. Apes were tested individually in these familiar dormitories buildings. Following testing, most apes were released back with their larger social group outside. Apes had ad libitum access to water and were never food deprived for testing. In addition to the food the apes could eat in their forest enclosures, they were fed a variety of fruits, vegetables, and other species-appropriate food 2-4 times daily. Subjects completed no more than one test session per day, and participating in all tests were voluntary: if the ape stopped participating, the session was halted.

\subsubsection{Subjects}

Ten bonobos ( 7 females and 3 males) participated in this study, ranging in age from 3 to 7 years of age (mean age $=4.2 \pm 0.4$ years; see Table 1 for all subject details) living at Lola ya Bonobo Sanctuary. Apes in African sanctuaries are typically born in the wild, and enter the sanctuary after being confiscated at an early age (approximately 2-3 years old) as a result of 
Table 1.

Subject characteristic and performance in Study 1.

\begin{tabular}{lcccc}
\hline Subject & Sex & Age & Learning trials & Probe trials: allocentric \\
\hline Kanaga & + & 2 & 0.93 & 1.00 \\
Kipolo & $\sigma^{7}$ & 5 & 0.85 & 0.67 \\
Kodoro & $\sigma^{7}$ & 3 & 0.85 & 1.00 \\
Lukuru & + & 4 & 0.64 & 0.83 \\
Masisi & + & 4 & 0.83 & 1.00 \\
Muanda & + & 6 & 0.69 & 0.33 \\
Sandoa & + & 3 & 0.96 & 0.83 \\
Shibombo & $\sigma^{7}$ & 3 & 0.94 & 1.00 \\
Tchilenge & + & 7 & 0.78 & 0.50 \\
Waka & + & 4 & 0.81 & 0.17 \\
Overall & & & 0.83 & 0.73 \\
\hline
\end{tabular}

Age estimate is listed in years. Learning trials indicates the proportion of correct choices across all learning trials. Probe trials indicates the proportion of test trials where subjects made an allocentric (spatial) response; they made an egocentric response on the remainder of trials.

the trade in apes for pets and bushmeat. Previous work indicates that sanctuary apes are psychologically healthy relative to other captive populations (Wobber \& Hare, 2011). As exact birth dates for sanctuary apes are generally unknown, we used age estimates made by sanctuary veterinarians at arrival (typically when the infants are between 1 and 3 years old), and adjusted these age estimates using measurements of weight and patterns of dental emergence according to known patterns of ape development (following the methods described in Wobber et al., 2010, 2013; Rosati \& Hare, 2012), a technique that is further validated using the known ages of individuals born in the sanctuaries. The apes had previously participated in a variety of cognitive testing involving making choices between options presented on tables (e.g., Wobber et al., 2013), but were naïve to the particular task here.

\subsubsection{General setup}

Apes were tested individually in a room in their familiar sleeping dormitory, with the experimenter and a caretaker present. They were presented with two identical and opaque overturned bowls $(24.5 \mathrm{~cm}$ diameter, $15 \mathrm{~cm}$ tall, and red in color) that were placed $2.5 \mathrm{~m}$ apart; food could be hidden under the bowls. The caretaker sat at the starting position with the bonobo in front 


\section{(1) Learning trials}
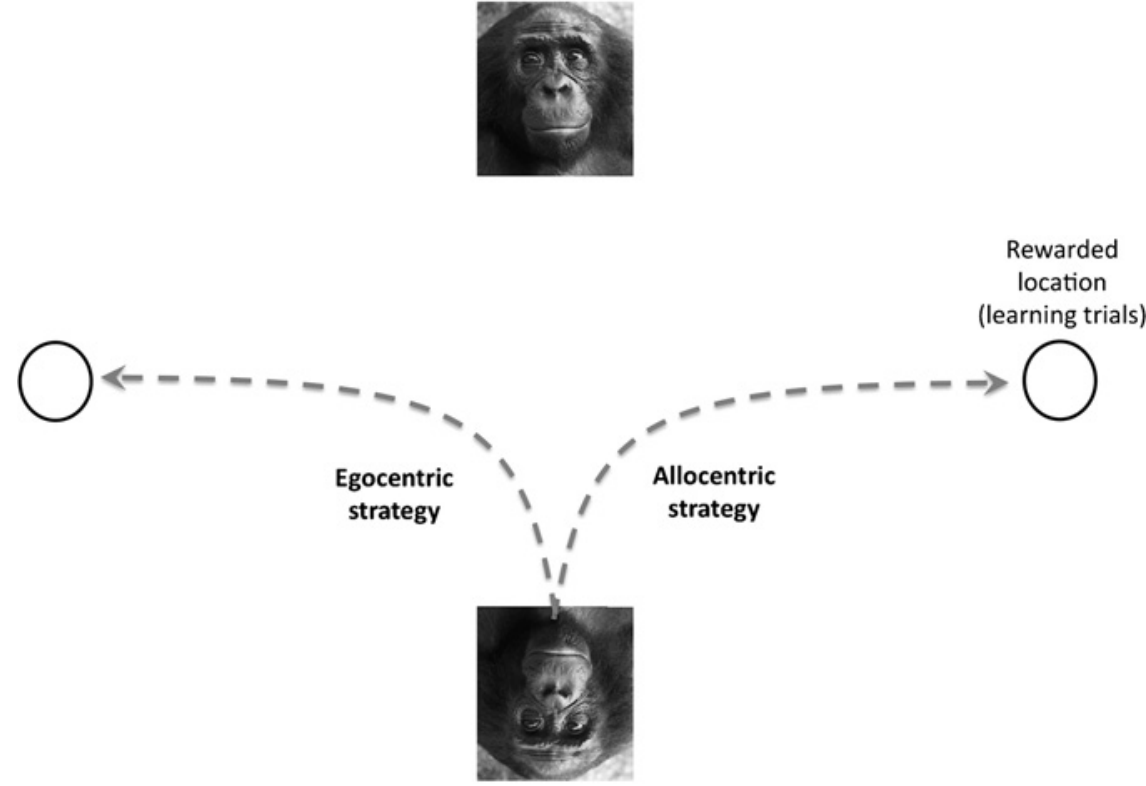

(2) Probe trials

Figure 1. Setup for place-response task (Study 1). In each session, apes first completed 12 learning trials in which one of two locations (both overturned bowls) was consistently baited. In the final probe trial, the apes' starting position was rotated $180^{\circ}$ so they faced the locations from a flipped perspective. Their responses therefore indicated if they had encoded the baited location from a viewer-dependent (egocentric) framework or a spatial (allocentric) framework. On all trials the caretaker centered the bonobo at their starting position, and the experimenter stood in the opposite position across the midline of the testing area.

of him, centering the bonobo approximately $1 \mathrm{~m}$ away from the midline of the two bowls (see Figure 1).

\subsubsection{Session and trial structure}

The bonobos completed 6 sessions total, consisting of three types of trials. All sessions were identical with the exception of the introductory trials in the first session (described below). A given bonobo was always tested in the same room, and the orientation of the setup in that room as well as the baited location in learning trials was kept identical across sessions, following the methods of previous work using this task in other species (Packard, 1996, 1999, 2009). In particular, the consistent setup across sessions made it possible to assess if the apes' spatial strategy changed over time, following 
previous work showing that rodents initially exhibit allocentric strategies but switch to egocentric strategies with increase repetition. The trial types in the sessions were as follows:

(1) Introduction trials (two trials, only in the first session): At the beginning of their first session, apes completed two introduction trials where they visibly saw the experimenter placing food under both bowls. Here, the caretaker directed the subject to the centered starting position while the experimenter baited the bowls (with bonobos typically sitting on their lap). The experimenter showed the ape two pieces of preferred food (apple), approached both bowls (in counterbalanced order), called the ape's name, and visibly placed a piece of food under each bowl while the ape watched. These trials therefore served to introduce the idea that the bowls could be baited, and allowed bonobos to successfully experience retrieving food from both. Once the experimenter finished baiting, she returned to a midline position across from the ape and looked straight ahead to avoid cuing the subject. After the experimenter said "start", the keeper released the bonobo so he or she could choose one of the bowls by approaching it. Bonobos had to approach both bowls to retrieve the food in order to proceed to the subsequent learning trials in that session; if bonobos who did not initially approach both locations on their own, the experimenter attracted their attention to the other bowl. These trials were not included in sessions two through six.

(2) Learning trials (12 in each session): These trials were similar to introductory trials, but only one bowl was baited. When the experimenter approached both bowls (in counterbalanced order within sessions), she placed her hand under the bowl while covering it with an occluder, such that the ape could not see which location had actually been baited with the food. If the subject chose incorrectly by approaching the empty bowl in learning trials, they could correct their choice (e.g., then approach the correct location) in order to equate reward experience across all subjects (following previous studies; Packard, 1996). After the bonobo retrieved the food, the caretaker attracted them back to the starting position.

(3) Probe trials (one trial at the end of each session): The procedure for probe trials was similar to that for learning trials. Prior to the trial, the caretaker walked with the ape to the opposite side of the bowls so that the ape started in the flipped position $\left(180^{\circ}\right)$ relative to the bowls and the larger spatial layout of the room. The trial then proceeded like learning trials, with two main exceptions. First, the experimenter baited both bowls (approaching 
them in counterbalanced order across sessions) to avoid biasing the subjects in subsequent trials. That is, the animal always was rewarded regardless of what strategy they exhibited. Second, subjects could only approach one bowl so there was no opportunity for self-correction; the caretaker attracted the bonobo away from the bowls after they made a choice.

\subsubsection{Coding and data analysis}

Choices were coded live by the experimenter. A coder blind to the correct side coded $20 \%$ of sessions from videotape for reliability; agreement was excellent (Cohen's kappa $=0.99$ ). Choice data were analyzed using two statistical approaches. The first approach used parametric statistics. For these analyses, mean proportions of choices for the correct location (in learning trials) and for allocentric responses (in probe trials) were calculated for each subject.

The second approach focused on the bonobos' responses to probe trials, the main measure of interest. This approach used generalized linear mixed models (GLMM) to analyze choices as a binary outcome variable, while accounting for correlation in responses due to repeated measures within subjects (Baayen, 2008). This analysis used the LME4 software package (Bates, 2010) in the statistics program $R$ ( $R$ Development Core Team, 2011). The advantage of using a GLMM model, compared to ANOVA, is that it allowed trial-by-trial modeling of responses, while also controlling for various covariates and random effects (such as subject). These analyses used likelihood ratio tests to compare fit across models incorporating different factors (Bolker et al., 2008). Across models, subject was included as a random factor, and predictors included sex (as a factor), age in years (as a covariate), session number (one through six) as a covariate, and proportion of correct learning trials in that session (as a covariate). This analysis therefore could examine the degree to which age, sex, and previous learning trial experience predicted the bonobos' responses on probe trials.

\subsection{Results and discussion}

The first analysis focused on the bonobos' performance in learning trials. Overall, bonobos chose correctly on $82.8 \pm 3.3 \%$ (mean \pm SE) of all learning trials, significantly above chance overall (one sample $t$-test: $t_{9}=9.91$, $p<0.001$; see Table 1 for subject means). Indeed, apes were above chance in selecting the correct location in learning trials across all six sessions (session 1: mean $\pm \mathrm{SE}=65.8 \pm 6.3 \%, t_{9}=2.53, p<0.05$; session 2: $75.0 \pm$ 
$5.0 \%, t_{9}=5.03, p=0.001 ;$ session $3: 75.0 \pm 7.8 \%, t_{9}=3.22, p=0.01$; session 4: $90.8 \pm 3.6 \%, t_{9}=11.31, p<0.001$; session 5: $93.3 \pm 2.7 \%$, $t_{9}=15.92, p<0.001$; session 6: $\left.96.7 \pm 1.4 \%, t_{9}=34.29, p<0.001\right)$. A repeated-measures ANOVA comparing learning trial performance across sessions revealed a main effect of session $\left(F_{5,45}=9.62, p<0.001\right)$ with a significant linear contrast $\left(F_{1,9}=30.18, p<0.001\right)$ indicting the bonobos' recall of the baited location improved with repeated exposure to the correct location. Pair-wise comparisons confirmed this: the apes' memory in session one was less accurate than that in sessions $4-6$, and session 2 performance was also lower than in session 6 (Bonferroni correction; $p<0.05$ for all significant cases). That is, the bonobos' performance in their last sessions was significantly better than in their initial sessions.

We next examined performance on probe trials. Bonobos made an allocentric response on $73.3 \pm 9.7 \%$ of trials, significantly above chance $\left(t_{9}=2.41\right.$, $p<0.05$; see Table 1 for subject means). Examining the performance of individual subjects revealed that seven of the ten subjects made predominantly allocentric choices (67-100\% allocentric choices), two subjects made predominantly egocentric choices (17-33\% egocentric choices), and one individual showed no overall preference (exhibiting both strategies equally across probe trials). A comparison of performance on learning compared to probe trials indicated that there was no significant difference between overall correct choices in the learning trials compared to allocentric choices in the probe trials (paired sample $t$-test: $t_{9}=1.09, p=0.30$, n.s.; see Figure 2a). That is, overall bonobos chose the allocentric option at levels comparable to their rate of success on learning choices. This suggests that they generalized their experience from the learning trials to predict that the food was located in the same spatial location in probe trials. Finally, while there was no relationship between an individual's performance in learning trials and allocentric choices in probe trials $\left(r_{\mathrm{p}}=0.47, p=0.17\right.$, n.s. $)$, there was a negative relationship between age and probe trial performance: older bonobos were less likely to make allocentric responses than younger apes $\left(r_{\mathrm{p}}=-0.65\right.$, $p<0.05$ ). The results from the GLMM, which modeled each individual probe trial choice as a binary outcome, generally confirmed these results. The first step was to fit a basic model including subject as a random factor, sex as a fixed factor, and proportion of correct learning trials as a covariate. Learning trial performance was included in the model to control for the possibility that the bonobos' performance in the immediately preceding session 

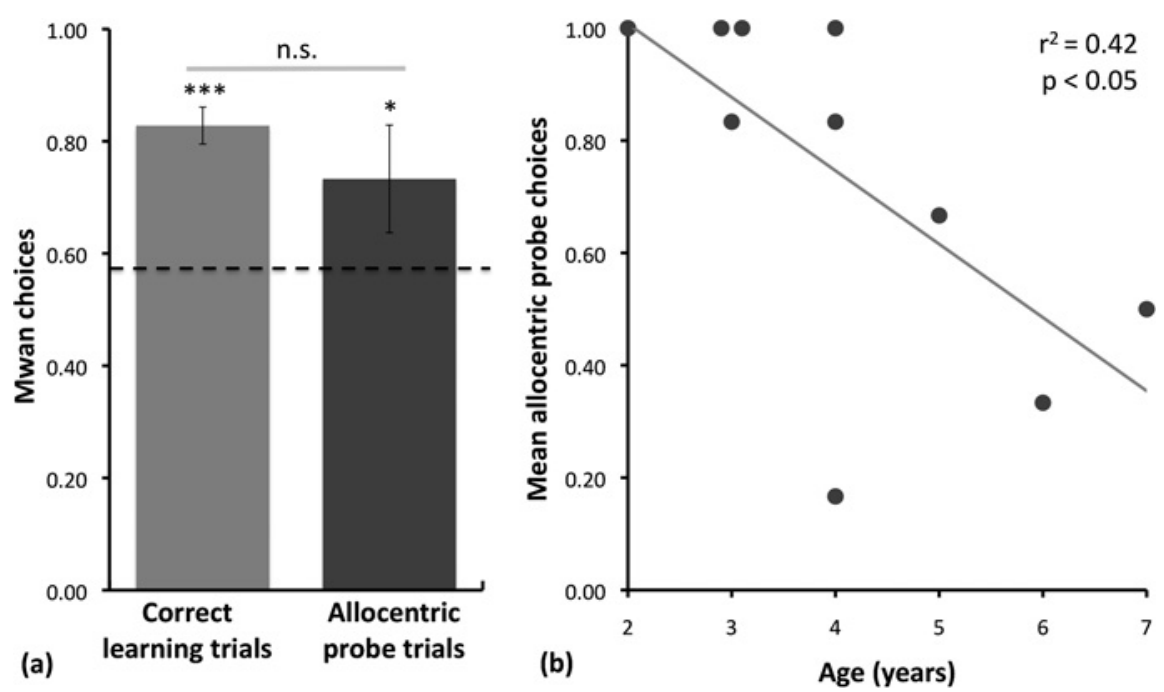

Figure 2. Results from place-response task (Study 1). (a) Bonobos' mean proportion choices for the correct location in learning trials, and for the allocentric location in probe trials. (b) Relationship between overall allocentric probe choices and the subject's age. Error bars indicate standard error. ${ }^{*} p<0.05,{ }^{* * *} p<0.001$.

predicted their probe choices (note that all bonobos had equal experience with the correct location regardless of their initial choice, as bonobos were allowed to self-correct following incorrect choices in the learning trials). In a second model, session number was included as a covariate to the more parsimonious basic model to assess whether apes showed any shifts in probe trial performance over time. Finally, in a third model age was also added as a covariate to assess any potential effects of cognitive development on the bonobos' performance. The full model revealed that age was a significant negative predictor on the apes' propensity to make an allocentric response (see Figure $2 b$ and Table 2 for parameters from the full model). However, there was no change in probe strategies over time. Moreover, whereas comparing the basic model to the second model including session number did not improve model fit ( $\chi^{2}=0.13$, $\mathrm{df}=1, p=0.72$, n.s. $)$, the third model also including age did show greater fit (model 2 versus model $3: \chi^{2}=3.89$, $\mathrm{df}=$ $1, p<0.05)$. This highlights the importance of age as a predictor of apes' allocentric responses.

Overall, these results indicate that the bonobos primarily used an allocentric spatial strategy in this context. The bonobos quickly learned the location 
Table 2.

Factors influencing the apes' likelihood for making an allocentric response on probe trials in Study 1.

\begin{tabular}{lrrrr}
\hline Factor & Estimate & SE & $Z$ & $p$ \\
\hline Sex & 1.363 & 1.302 & 1.047 & 0.30 \\
Age & -0.824 & 0.406 & -2.031 & $<0.05^{*}$ \\
Session & -0.036 & 0.284 & -0.127 & 0.90 \\
Correct learning trials & -0.100 & 2.750 & -0.036 & 0.97 \\
\hline
\end{tabular}

The full generalized linear mixed model assessed the influence of the following factors: sex (female as baseline), age (in years), session number (1-6) and learning trial performance (proportion correct in the preceding learning trials that session).

* Significant value.

that was baited in learning trials, showing a preference for that location from the first session. When the bonobos' orientation was flipped so they approached the bowls from the opposite direction, they generalized their experience in learning trials to consistently select the allocentric location. That is, they approached the same location in space that had been baited previously, rather than using a viewer-dependent frame of reference. This generalization did not change across sessions, indicating the repeated experience with the correct location on learning trials did not shift the bonobos from using an allocentric strategy towards an egocentric strategy, as has been previously demonstrated in other animals (Packard, 2009). However, probe trial performance was negatively related to age - younger bonobos were more likely to make allocentric responses on probe trials than were older bonobos - and a model including age as a factor was a better fit in the GLMM analysis. It is important to point out, however, that the age range and sample size in this study were small. Thus, the second study examined spatial cognition in a large sample of bonobos ranging from infants to adults. In addition, this study used a different task that involved choices between three options presented on a smaller scale to examine if the bonobos' allocentric strategies were stable across contexts.

\section{Study 2: spatial relations task}

The second study examined how bonobos process spatial relations by presenting them with two tables with identical arrays of three overturned opaque cups on top, following the basic procedure from (Haun et al., 2006a). Apes 


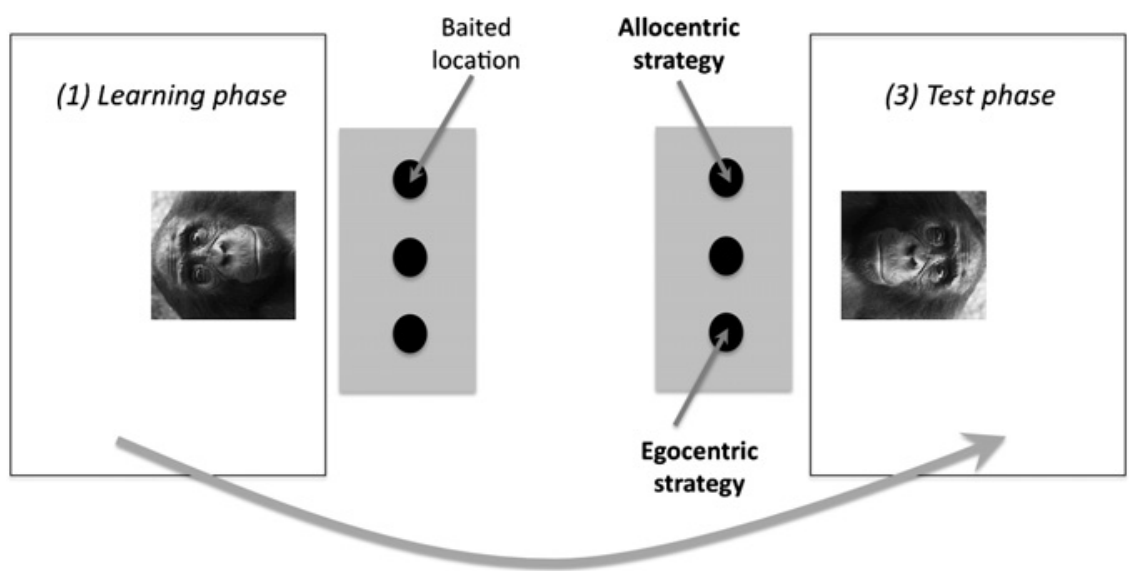

(2) Rotate positions

Figure 3. Setup for relations task (Study 2). Apes first completed a learning phase in which one location (always either the left or right side) was consistently baited. After apes met a learning criterion indicating they consistently chose the correct side, they moved $180^{\circ}$ into a new room for the test phase. In the last 10 trials, apes faced an identical table from a flipped perspective to assess if they had encoded the baited location from a viewer-dependent (egocentric) framework or a spatial (allocentric) framework. The experimenter sat centered behind the table at both locations.

first made choices at one of the tables and learned that one of the outer cups (left or right) always contained food. After demonstrating that they would consistently pick that cup, apes moved to the second table where apes' orientation was flipped $180^{\circ}$ (see Figure 3). In this second test phase, bonobos could use either use an allocentric strategy (choosing the cup with the same orientation in the broader spatial layout), use an egocentric strategy (choosing the cup with the same relation to their own body), or fail to generalize their previous experience by choosing the center location.

While this study followed the same basic procedure as that by Haun and colleagues (2006a), there were some adjustments to the methods to test a larger age range of subjects (in particular, younger bonobos). First, each individual only completed one session where one location was baited, to see how this experience generalized in the test phase, whereas in the previous study apes were trained on all possible locations across three different sessions. Second, bonobos initially completed several types of warm-up trials to ensure they were proficient with choosing all the various cup locations, accounting for the fact that the infants were less familiar with these kinds of table-based tasks than are older apes. Finally, we did not include the initial 
baseline trials used by Haun and colleagues (2006), used there to detect if the apes had some initial bias for one of the locations in the absence of any relevant experience in the study. As apes in the current study had to demonstrate proficiency with choosing all three locations in the warm-up phase, the current analyses therefore focused only on mean choices in the test phase, and did not involve a difference score measuring change between the baseline and test phase.

\subsection{Methods}

\subsubsection{Subjects}

Nineteen bonobos ( 9 females and 10 males) from the same population of bonobos living at Lola ya Bonobo Sanctuary participated in this study. Individuals ranged from 3 to 13 years of age (mean age $=6.6 \pm 0.7$ years; see Table 3 for all subject characteristics). Seven individuals had participated in Study 1 approximately 1 year previously.

\subsubsection{General setup}

Apes were tested in two rooms that faced each other across a hallway. The basic setup consisted of two tables (each $80 \mathrm{~cm}$ wide, $40 \mathrm{~cm}$ deep, $40 \mathrm{~cm}$ high) with sliding tops that allowed the objects on the tables to be set up and then moved into the reach of the apes. Each table was placed in the hallway and facing one of the rooms. An identical array of three overturned cups (diameter $5 \mathrm{~cm}$, height $10 \mathrm{~cm}$ ) was placed on each table. During testing, apes sat in one of the rooms facing the array of three cups, and the experimenter sat across the table from the apes, separated by bars. Apes were tested individually; adults were alone in the test rooms, whereas infants were tested with the human caretaker present to ensure that they were comfortable. The caretaker sat at the back of the room behind the bonobo, and did not interact with the ape or provide any cues. Apes always began the study in one room (for the learning phase) facing one of the tables, and then moved to the second room (for the test phase) via an overhead tunnel connecting them (juvenile and adult bonobos), or by being walked across the hallway by their caretaker (infant bonobos). The width of the hallway between the learning phase room and the testing phase room (as well as the overhead tunnel that adults utilized) was $2 \mathrm{~m}$. Thus, the two tables were also $2 \mathrm{~m}$ apart.

In contrast to the first study, this setup made it possible to disentangle whether some apes do not generalize their initial learning to the test phase, versus whether some bonobos exhibit a mixed strategy involving both 
Table 3.

Subject characteristic and performance in Study 2.

\begin{tabular}{|c|c|c|c|c|c|}
\hline \multirow[t]{2}{*}{ Subject } & \multirow[t]{2}{*}{ Sex } & \multirow[t]{2}{*}{ Age } & \multirow[t]{2}{*}{ Learning trials } & \multicolumn{2}{|c|}{ Test trials } \\
\hline & & & & Allocentric & Egocentric \\
\hline Dilolo & $0^{7}$ & 10 & 30 & 0.0 & 0.8 \\
\hline Fizi & $\sigma^{x}$ & 11 & 21 & 0.0 & 1.0 \\
\hline Kalina & $q$ & 13 & 11 & 0.0 & 0.0 \\
\hline Kanaga* & q & 3 & 17 & 0.2 & 0.0 \\
\hline Kasongo & $0^{7}$ & 9 & 29 & 0.0 & 0.9 \\
\hline Katako & q & 7 & 13 & 0.0 & 0.9 \\
\hline Kikwit & $0^{7}$ & 12 & 20 & 0.0 & 0.9 \\
\hline Kindu & q & 3 & 23 & 0.0 & 0.4 \\
\hline Kodoro* & $\sigma^{7}$ & 4 & 20 & 0.0 & 0.9 \\
\hline Lomako & $0^{x}$ & 4 & 21 & 0.0 & 1.0 \\
\hline Lukuru* & q & 5 & 12 & 0.0 & 0.9 \\
\hline Mabali & $0^{7}$ & 8 & 19 & 0.0 & 0.1 \\
\hline Maliaka & q & 4 & 22 & 0.0 & 1.0 \\
\hline Masisi* & q & 5 & 18 & 0.0 & 0.9 \\
\hline Matadi & $0^{7}$ & 11 & 31 & 0.0 & 1.0 \\
\hline Oshwe & $\sigma^{7}$ & 3 & 18 & 0.0 & 1.0 \\
\hline Sandoa* & q & 4 & 12 & 0.0 & 1.0 \\
\hline Shibombo* & $0^{x}$ & 4 & 27 & 0.0 & 0.4 \\
\hline Waka* & q & 5 & 30 & 0.0 & 0.0 \\
\hline Overall & & & 20.7 & 0.01 & 0.69 \\
\hline
\end{tabular}

Age estimate is listed in years. Learning trials indicates the total number of learning trials that the subject completed to meet criterion. Test trial columns indicate the proportion of allocentric and egocentric choices in the test phase; the remainder of choices were for the center option. ${ }^{*}$ Subjects that previously participated in Study 1.

egocentric and some allocentric choices, due to the presence of the center location. Moreover, these methods could be used with older apes as the experimenter was in a different room than the subject.

\subsubsection{Session and trial structure}

In the initial learning phase, apes learned that one cup (always one of the outer cups, left or right side assignment counterbalanced across subjects) provided food. Here, apes completed four types of trials, including various warm-up trials to familiarize the apes to the setup, as well as the learning trials where apes learned that one particular cup was consistently baited. Across all trials only one cup was baited with food on a given trial. After meeting a criterion indicating they consistently chose the baited location, apes were 
switched $180^{\circ}$ to the other table, faced with an identical array of cups for the test phase. In those trials all three cups were baited to examine how the apes' generalized their previous learning to the new array. All trials were completed as part of the same session. The order of trials was as follows:

(1) Visible food trials (one per cup location in randomized order, three trials total): In the first part of the learning phase, bonobos completed a basic introduction to making choices on the table and familiarize them to the procedure (as some of the infant apes were naïve to this test setup). In particular, food was placed on top of one of the cups and thus was visible at the time the ape made their choice. These trials introduced the bonobos to the procedure of choosing cups to get the food, and were repeated if the bonobo initially chose incorrectly to ensure that all bonobos were proficient with this basic skill.

(2) Hidden food trials (two per cup location in randomized order, six trials total): Next, apes saw the experimenter place the food under the correct cup, but it was hidden under the cup at the time of choice. These trials introduced the idea of choosing concealed food, and were also repeated if the bonobo initially chose incorrectly. Thus, these warm-up trials ensured that bonobos had to demonstrate an ability to correctly choose hidden food items before proceeding to the learning phase.

(3) Help trials (four trials, always involving the same cup as in the learning trials): After the warm-up phase was completed, apes learned that only one of the cups (left or right) consistently contained food. In the four initial help trials, the experimenter placed the food visibly under the baited side cup. These trials were similar to the previous hidden food trials, except that only the one cup was baited; in addition, if the ape chose incorrectly, they were shown that their choice was empty as well as the location of the correct choice, without being allowed to repeat the trial to obtain the food.

(4) Learning trials (as main trials necessary to meet criterion, up to 32 trials total): In the last type of trial, the experimenter hid the food under the baited cup behind an occluder (the same side as had been baited in the help trials). The experimenter touched all three cups in a counterbalanced order behind the occluder so as to not give any auditory or movement cues about the location of the food. As in help trials, if the ape chose incorrectly, they were shown that their choice was empty as well as the location of the food, but were not allowed to correct their choice. Apes had to choose the correct location in 10 of the previous 12 trials to meet the criterion and move onto 
the test phase. They had to meet this criterion within 32 trials or the session was halted without proceeding to the test phase. Three bonobos did not meet this criterion within 32 trials, so they were given a break of several days and then repeated the session (all three then met the criterion). The limit was set after relatively few trials to ensure that the infant bonobos would remain food-motivated to continue for the duration of the session, as they would become disinterested if the session continued for an extended period.

(5) Test trials (10 in total, all three locations baited): After meeting the learning criterion, apes were switched $180^{\circ}$ to the other table, faced with an identical array of cups during the test phase. As in learning trials, the experimenter baited the cups behind an occluder and touched all three cups in counterbalanced order to avoid giving any clues to the food's location. In contrast to the learning trials, however, here the experimenter baited all three locations so as to avoid biasing the ape's choices on subsequent trials. Once the ape made a choice, they received the food from their chosen cup and were not shown the contents of the other cups. This allowed us to assess if the apes were using an allocentric strategy (chose the cup in the same spatial direction as in the learning trials), and egocentric strategy (chose the same cup from their own perspective, left or right), or did not generalize their previous learning to this new context. The side of the hallway assigned as the learning versus test room was counterbalanced across subjects to ensure that any external spatial cues were counterbalanced.

\subsubsection{Coding and data analysis}

Choices were coded live by the experimenter. A coder blind to the correct side coded $20 \%$ of sessions from videotape for reliability; agreement was $100 \%$. Choice data was analyzed using the same approaches as in the first study. The first approach used parametric statistics to assess mean proportions of choices for the correct location (in learning trials) and for allocentric versus egocentric responses (in test trials) for each subject. The second approach used generalized linear mixed models (GLMM) to analyze test choices on a trial-by-trial basis.

\subsection{Results and discussion}

The first analysis focused on the bonobos' performance in learning trials. Bonobos took an average of $20.7 \pm 1.5$ learning trials to reach the criterion of choosing the last ten of twelve trials correctly. Overall, they chose correctly on $66.1 \pm 3.2 \%$ of all learning trials, significantly above chance overall (with 
chance at 33.3\%; one sample $t$-test: $t_{18}=10.38, p<0.001$; see Table 3 for subject means). A univariate GLM with sex as a between-subjects factor and age as a covariate revealed that female bonobos reached criterion in fewer trials than did males (females requires an average of $17.6 \pm 2.1$ trials compared to $23.6 \pm 1.5$ trials for males; $F_{1,16}=4.66, p<0.05$ ). However, there was no effect of age on the number of learning trials $\left(F_{1,16}=0.03\right.$, $p=0.88$, n.s.), indicating that younger and older bonobos learned the baited location at similar rates.

The next analysis examined the bonobos' performance on test trials, where they faced a set of cups from a flipped perspective (see Figure 4a and Table 3 for subject means). Across all subjects, bonobos made an allocentric responses on only $1.1 \pm 1.1 \%$ of trials, significantly below chance (at $\left.33.3 \% ; t_{18}=-30.66, p<0.001\right)$. In contrast, bonobos showed a clear pattern of making egocentric generalizations, choosing that option on $69.0 \pm$ $9.0 \%$ of trials, significantly above chance $\left(t_{18}=3.93, p=0.001\right.$; see Table 3 for subject means). A comparison of egocentric versus allocentric responses indicated that apes were more likely to use an egocentric strategy $\left(t_{18}=-7.12, p<0.001\right)$. Apes chose the middle option $30.0 \pm 8.7 \%$, at chance levels $\left(t_{19}=-0.39, p=0.71, \mathrm{n} . \mathrm{s}\right.$.); these responses represent fail-

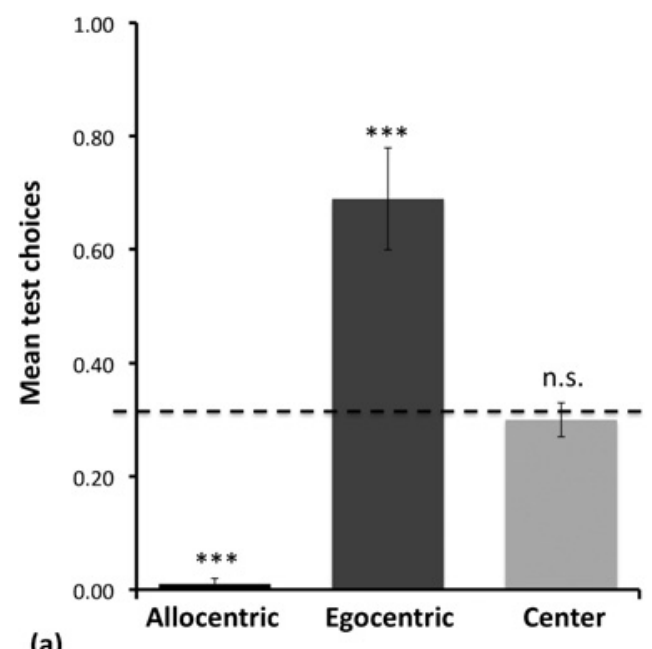

(a)

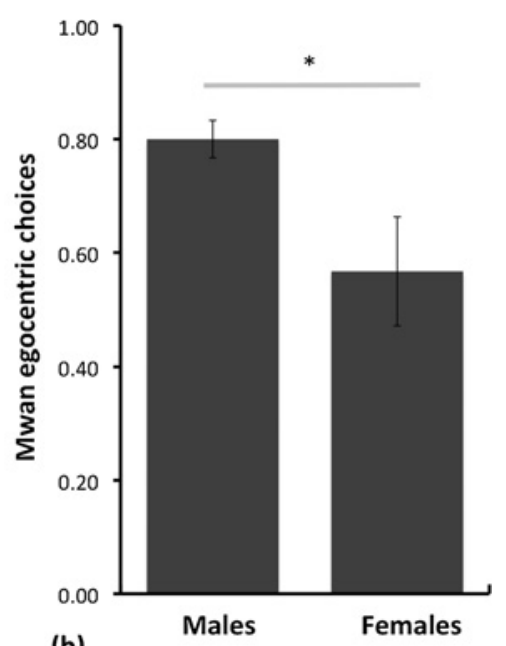

(b)

Figure 4. Results from the relations task (Study 2). (a) Bonobos' mean proportion choices in the test phase for the allocentric option, the egocentric option, and the middle option. (b) Breakdown of egocentric choices in test phase by the subjects' sex. Error bars indicate standard error. ${ }^{*} p<0.05,{ }^{* * *} p<0.001$. 
ures to generalize their previous experience to the test phase. Examining the performance of individual subjects revealed that 13 of the 19 subjects made predominantly egocentric choices (80-100\% egocentric choices), but no individuals preferred the allocentric option. Indeed, the remaining six individual bonobos predominantly chose the center location, suggesting a lack of generalization (they made $60-100 \%$ of choices for the center cup during the test phase). Overall, this suggests that bonobos tended to generalize their experience from the learning trials to predict that the food was located in the locations with the same relationship to their own body (e.g., they encoded the baited location based on whether it was to their own left or right, not based on external spatial cues). Finally, the number of learning trials need to reach criterion was not correlated with either the proportion of egocentric test responses that bonobo exhibited in the test phase $\left(r_{\mathrm{p}}=0.01, p=0.99\right.$, n.s.), nor allocentric test responses $\left(r_{\mathrm{p}}=-0.14, p=0.56\right.$, n.s.).

The GLMM analysis modeled each test trial choice in terms of whether or not the bonobo made an egocentric response (as that was the dominant type of response). The first step fit a basic model including subject as a random factor; sex as a fixed factor; and number of learning trials necessary to reach criteria as a covariate to assess whether the bonobos' learning patterns predicted their test choices. In a second model, test trial number was included as a covariate to the more parsimonious basic model to ascertain whether apes showed any shifts in their test preferences over time. Finally, in a third model age was also added as a covariate to assess any potential effects of cognitive development on the bonobos' performance. The full model revealed that male bonobos showed more egocentric choices than females (at $80.0 \pm 9.7 \%$ for males, but only $56.7 \pm 15.4 \%$ for females; see Figure $4 \mathrm{~b}$ ). This was the case even when controlling for their differential learning patterns in the learning phase, as learning trials to criterion was included as a covariate. In addition, apes' egocentric choices increased over the course of the ten test trials (see Table 4 for parameters from the full model). Moreover, while the second model including trial number did improve model fit in comparison to the basic model (model 1 versus model 2: $\chi^{2}=4.59, \mathrm{df}=1$, $p<0.05$ ), the third model including age did not improve fit in terms of predicting whether apes' made an allocentric response (model 2 versus model 3 : $\chi^{2}=0.94, \mathrm{df}=1, p=0.33$, n.s.). Thus, there was no effect of age on the bonobos' patterns of choices in the test phase. 


\section{Table 4.}

Factors influencing the apes' likelihood for making an egocentric response on test trials in Study 2.

\begin{tabular}{lrrrc}
\hline Factor & Estimate & SE & $Z$ & $p$ \\
\hline Sex & 4.422 & 2.219 & 1.993 & $<0.05^{*}$ \\
Age & -0.286 & 0.294 & -0.972 & 0.33 \\
Test trial number & 0.202 & 0.096 & 2.099 & $<0.05^{*}$ \\
Learning trials to criterion & -0.163 & 0.163 & -1.001 & 0.32 \\
\hline
\end{tabular}

The full generalized linear mixed model assessed the influence of the following factors: sex (female as baseline), age (in years), trial number (1-10) and number of learning trials necessary to reach criterion in preceding learning phase.

* Significant value.

Overall, these results indicate that the bonobos primarily used an egocentric strategy in this context. That is, after the bonobos learned which cup was baited in the learning trials, the test trials revealed that they had encoded this location in terms of a viewer-dependent framework. Indeed, bonobos rarely made an allocentric response: in the vast majority of trials they did not make an egocentric response, they chose the middle cup (suggesting lack of generalization from the previous trials). Finally, The GLMM analysis indicated that bonobos tended to increase their choices for the egocentric location over the course of the ten test trials, but this reflected a decrease in center cup choices, not a change in their likelihood of making an allocentric response. This analysis further indicated that male bonobos were more likely to make this egocentric generalization; females in contrast showed greater rates of choosing the middle cup, suggesting those individuals did not generalize their previous experience to the test phase. One possibility is that males' slower learning rates in the learning phase meant that they in fact received more feedback about the correct location, and therefore more likely to generalize this experience in the test phase (although, the number of learning trials apes took to reach criterion was not a significant predictor of their probe trial performance). Regardless, no bonobo of either sex exhibited a bias towards an allocentric strategy in the test trials; in fact there was only one individual who ever chose it.

\section{General discussion}

The current studies had two major results. First, across two commonly used but distinct tasks to assess spatial frameworks, bonobos were able to use 
both egocentric and allocentric strategies. In the first study, apes experienced that one of two locations in a larger spatial area was consistently baited with food, and then their strategy was assess by flipping their orientation towards the testing arena. Here, the bonobos showed allocentric inferences, tending to go to the same location (rather than making the same egocentric body movement) when searching for the food. In the second study, apes learned that food was delivered under one of three cups. When their orientation was flipped and they faced an identical set of three cups, however, apes tended to show an egocentric strategy - choosing the cup with the same position relative to their own body (e.g., to the left or right) but not in the same relationship to the external environment. Second, the results from these studies provide little evidence for systematic developmental change in the frames of reference that bonobos use. There was some suggestion of increases in use of egocentric frame in Study 1, but that study had a small sample size. In contrast, the results from Study 2 with a larger sample size did not reveal any age-related change in strategies. Overall, these results indicate that bonobos can used both allocentric and egocentric frameworks, but there was not consistent evidence for age-related shifts in their use of these strategies.

One major question raised by these results is why the bonobos exhibited different strategies in the place-response task versus the spatial relations task. Importantly, studies of multiple memory systems suggest that many diverse contextual factors can bias organisms toward the use a given strategy in dualsolution tasks that can be solved in either fashion (Packard \& Goodman, 2013). For example, one factor that can shift control of behavior between hippocampal-based allocentric strategies and basal-ganglia-based egocentric strategies is the amount of training involved in the task. Rats that experience extended periods of training in the plus-maze task show a switch in their response to probe trials over time: although initial responses tend to be allocentric, over time rats show more egocentric choices (Packard, 1996, 1999). However, training effects are unlikely to have driven the difference between the two studies presented here for two reasons. First, bonobos exhibited allocentric strategies in the place-response task, which actually involved more training over a longer time period (e.g., several sessions across multiple days) compared to the spatial relations task (which took place in one day). Second, there was no consistent evidence for shifts from allocentric to egocentric strategies in either task: in Study 1 there was no change in probe responses 
across days, and an increase in egocentric responses in Study 2 was due to a decrease in center-cup responses, not a shift from allocentric responses.

Another major factor that appears to influence animals' performance in the plus maze concerns the characteristics of the external environment (see Packard \& Goodman, 2013 for a review). In particular, an external environment with many varied visual cues and landmarks tends to bias animals towards using an allocentric strategy, likely because the presence of these landmarks fosters the formation of a cognitive map of the surrounds. In contrast, a more homogenous external environment with limited visual cues appears to bias animals towards an egocentric strategy based on their own movements. Similarly, the presence or absence of salient visual landmarks can bias the strategies that infants and young children exhibit in a variety of spatial tasks (Acredolo, 1978, 1979; Acredolo \& Evans, 1980; Newcombe \& Huttenlocher, 2006). However, differences in the external environment are also not a likely explanation for the current results, as the bonobos were either tested in the same building for both tasks, or in similarly constructed buildings (depending on the group the bonobo lived in at the sanctuary at the time of testing). These buildings all had similar visual features that could potentially serve as landmarks (e.g., windows serving as light sources, the presence of bars versus tiles on the walls, a hallway for humans to move through the building), and were fairly uniform rooms. However, variation in the external environment might be one factor driving the difference between the results in the spatial relations task (Study 2), where the bonobos exhibited egocentric strategies, and the results of a previous study in which apes exhibited primarily allocentric strategies (Haun et al., 2006a). For example, there may be differences in the visual cues across the testing facilities in these two studies, with more possible visual landmarks in the previous study. Additionally, one important difference between the two studies concerns the position of the experimenter relative to the cup arrays. In particular, in Study 2 the experimenter could not serve as a landmark as she was always centered relative to the cup array, sitting behind the table to administer the task (see Figure 3). However, in the previous study the experimenter sat to one side of the testing tables, and thus potentially could have served as a consistent landmark (e.g., the experimenter was physically closer to one side of the testing tables; see Figure 3 in Haun et al., 2006a). Thus, the presence of the human might have biased the apes towards using an allocentric strategy in that experiment. 
One noticeable difference between the place-response task used in Study 1 and the spatial relations task used in Study 2 is the size of testing 'arena'. Namely, bonobos had to make choices concerning locations that were $2.5 \mathrm{~m}$ apart in the first study, but chose between an array of cups on a table less than a meter total in width in the second study. Importantly, research using reorientation paradigms indicates that both children and adults utilize landmark-dependent allocentric strategies more often when faced with locating a target in a large space than in a smaller but otherwise-identical space (Learmonth et al., 2008; Ratliff \& Newcombe, 2008). One reason is that landmarks are more distal and salient in larger spaces, and therefore more useful cues for solving spatial problems. For example, in the current studies the positions of any available landmarks in the testing rooms might have been more relevant when the bonobos were making decisions between locations separated at larger distances in Study 1, than when they chose between the array of cups on the table in Study 2. This difference in size between the two studies also meant that the type of response the apes made to indicate their choice differed across the two studies. In particular, apes had to approach the locations by moving with their entire body over a distance of several meters in the place-response task, whereas in the spatial relations task the apes sat in front of a table and were within arms-reach of all the locations when making their choices. Some of the research on spatial strategies in children suggests that room size impacts strategies not only because of the relative salience of landmarks, but also due to how children can move around in the space. When children are tested in a room of a given size, children who are able to move throughout that room's space are more likely to use landmark information than are children who have identical visual access to the landmarks, but whose movements are confined to a smaller portion of the room (for example, if a smaller portion of the room is delineated by a line on the floor and children are asked to remain inside those lines; Learmonth et al., 2008). Notably, influence of room size or movement are not important factors in theories that focus on the role of language in spatial cognition (HermerVazquez et al., 1999; Wang \& Spelke, 2002; Majid et al., 2004; Haun et al., 2006a; Lee \& Spelke, 2010). In contrast, these types of contextual factors are thought to be important in adaptive-combination theories of spatial cognition (Newcombe \& Ratliff, 2007) in which the likelihood of using strategies is dependent on how different sources of information is weighted across different 
situations, for example due to the salience or usefulness of different sources of spatial information in particular environment.

Another issue raised by these results concerns why the bonobos did not exhibit age-related changes in spatial strategies. In particular, while there was some suggestion of increases in use of egocentric frame in Study 1, the smaller sample size in that study makes it difficult to assess how meaningful this age-related difference is. In contrast, the results from Study 2 with a larger sample size did not reveal any age-related change in strategies. Importantly, the sample size in that study was sufficient to allow detection of a sex difference in learning and generalization (e.g., females were quicker to learn which cup was baited in the learning phase, but then less likely to generalize this learning to produce either strategy in the test phase), but there was no indication that older and younger bonobos differed in their initial learning or in responses in the test phase. Thus, while developmental shifts in cognition are important in generating mature spatial competency in humans (Newcombe \& Huttenlocher, 2006), the current studies provide little evidence that bonobos' spatial frameworks exhibit any age-related change.

Notably, this aligns with results of previous research which suggest that chimpanzees show greater changes in cognitive skills across development than do bonobos (Wobber et al., 2010, 2013; Rosati \& Hare, 2012; Rosati et al., in press). For example, one study examined apes' spatial memory skills in a naturalistic foraging task in which animals had to recall the locations of many hidden rewards in a large-scale space (Rosati \& Hare, 2012). Even though chimpanzees and bonobos are very closely related, their developmental trajectories and mature levels of performance were quite different in this task: the bonobos exhibited no developmental shifts in accuracy of recall between infancy and sub-adulthood, whereas chimpanzees exhibit substantial improvement in the same age range and therefore outperformed bonobos as older ages. Thus, one possibility is that chimpanzees may in fact show shifts in spatial frameworks in the tasks used here with bonobos. For example, chimpanzees' improved performance in the foraging task may have been due to an overall increased reliance on allocentric frameworks that are more useful in a natural context with many salient, distal landmarks. Furthermore, some evidence suggests that primate species can differ in the cognitive strategies they use in these types of tasks: more frugivorous lemur species are more likely to use allocentric encoding compared to more folivorous lemur 
species (Rosati et al., 2014). Consequently, future studies should also examine whether chimpanzees show similar patterns compared to bonobos, or exhibit age-related changes in use of spatial frameworks.

What do these results mean for claims about human-unique spatial cognition? First, bonobos are able to use both egocentric and allocentric frameworks, which does not support claims that either allocentric (Haun et al., 2006a) or egocentric (Wang \& Spelke, 2002) frameworks alone are phylogenetically ancient. It also suggests that language is not necessary to flexibly utilize both types of frameworks. Rather, this supports views suggesting that parallel memory systems support nonhuman and human behavior (Burgess, 2006; Newcombe \& Huttenlocher, 2006). These results also indicate that some types of developmental change in spatial cognition seen in humans may not be present in bonobos. This suggests that differences in human and nonhuman patterns of development could result in human-unique cognitive capacities in adulthood. However, differences in the developmental trajectories of chimpanzees and bonobos suggests that these species might in fact provide contrasting views of ancestral developmental traits in humans, for the developmental trajectories and mature levels of performance in chimpanzees and bonobos differ for other spatial skills (Rosati \& Hare, 2012). Thus, models of the evolution of human cognitive development, including theories about the role of language in shaping spatial cognition, should integrate data on the development of both chimpanzees and bonobos in order to draw strong inferences about what traits are unique to our lineage.

\section{Acknowledgements}

Thanks to Brian Hare for advice on study design, Laura Lewis for assistance with coding and Felix Warneken for comments on a previous version of the manuscript. I also thank Claudine Andre, Dominique Morel, Valery Dhanani, Fanny Mehl and Pierrot Mbonzo and the animal caretakers for their support of this research at Lola ya Bonobo, in collaboration with the Ministry of Research and the Ministry of Environment in the Democratic Republic of Congo. This work was supported in by a L.S.B. Leakey Grant.

\section{References}

Acredolo, L.P. (1978). Development of spatial orientation in infancy. - Dev. Psychol. 14: 224-234. 
Acredolo, L.P. (1979). Laboratory versus home: the effect of environment on the 9-month-old infant's choice of spatial reference system. - Dev. Psychol. 15: 666-667.

Acredolo, L.P. \& Evans, D. (1980). Developmental changes in the effects of landmarks on infant spatial behavior. - Dev. Psychol. 16: 312-318.

Albiach-Serrano, A., Call, J. \& Barth, J. (2010). Great apes track hidden objects after changes in the objects' position and in subject's orientation. — Am. J. Primatol. 72: 349-359.

Baayen, R.H. (2008). Analyzing linguistic data. A practical introduction to statistics. Cambridge University Press, Cambridge, MA.

Bates, D. (2010). The LME4 package: linear mixed-effects models using S4 classes. Available online at http://www.R-project.org.

Bolker, B.M., Brooks, M.E., Clark, C.J., Geange, S.W., Poulsen, J.R., Stevens, M.H.H. \& White, J.S.S. (2008). Generalized linear mixed models: a practical guide for ecology and evolution. - Trends Ecol. Evol. 24: 127-135.

Brown, P. \& Levinson, S. (1992). 'Left' and 'right' in Tenejapa: investigating a linguistic conceptual gap. - Z. Phonetik Sprachwissensch. Kommunikationsforsch. 45: 590-611.

Brown, P. \& Levinson, S.C. (2000). Frames of spatial reference and their acquisition in Tenejapan Tzeltal. - In: Culture, thought, and development (Nucci, L., Saxe, G. \& Turiel, E., eds). Lawrence Erlbaum, Mahwah, NJ, p. 167-197.

Burgess, N. (2006). Spatial memory: how egocentric and allocentric combine. - Trends Cogn. Sci. 10: 551-557.

Burgess, N. (2008). Spatial cognition and the brain. - Ann. NY Acad. Sci. 1124: 77-97.

Cheng, K. (1986). A purely geometric module in the rat's spatial representation. - Cognition 23: $149-178$.

Gallistel, C.R. (1990). The organization of learning. - Bradford Books/MIT Press, Cambridge, MA.

Gentner, D. (2007). Spatial cognition in apes and humans. — Trends Cogn. Sci. 11: 192-194.

Hare, B. (2007). From nonhuman to human mind: what changed and why. - Curr. Dir. Psychol. Sci. 16: 60-64.

Hare, B., Wobber, V. \& Wrangam, R. (2012). The self-domestication hypothesis: evolution of bonobo psychology is due to selection against aggression. - Anim. Behav. 83: 573-585.

Haun, D.B.M., Rapold, C.J., Call, J., Janzen, G. \& Levinson, S.C. (2006a). Cognitive cladistics and cultural override in Hominid spatial cognition. - Proc. Natl. Acad. Sci. USA 103: $17568-17573$.

Haun, D.B.M., Call, J., Janzen, G. \& Levinson, S.C. (2006b). Evolutionary psychology of spatial representations in the Hominidae. - Curr. Biol. 16: 1736-1740.

Hermer, L. \& Spelke, E. (1994). A geometric process for spatial reorientation in young children. - Nature 370: 57-59.

Hermer-Vazquez, L., Spelke, E. \& Katnelson, A.S. (1999). Sources of flexibility in human cognition: dual-task studies of space and language. - Cogn. Psychol. 39: 3-36.

Hermer-Vazquez, L., Moffet, A. \& Munkholm, P. (2001). Language, space, and the development of cognitive flexibility in humans: the case of two spatial memory tasks. Cognition 79: 263-299. 
Herrmann, E., Hare, B., Call, J. \& Tomasello, M. (2010). Differences in the cognitive skills of bonobos and chimpanzees. - PLoS One 5: e12438.

Hoffman, M.L. \& Beran, M.J. (2006). Chimpanzees (Pan troglodytes) remember the location of a hidden food item after altering their orientation to a spatial array. - J. Comp. Psychol. 120: 389-393.

Hribar, A. \& Call, J. (2011). Great apes use landmark cues over spatial relations to find hidden food. - Anim. Cogn. 14: 623-635.

Hribar, A., Haun, D. \& Call, J. (2011). Great apes' strategies to map spatial relations. Anim. Cogn. 14: 511-523.

Janson, C.H. (1998). Experimental evidence for spatial memory in wild brown capuchin monkeys (Cebus apella). - Anim. Behav. 55: 1229-1243.

Janson, C.H. (2007). Experimental evidence for route integration and strategic planning in wild capuchin monkeys. - Anim. Cogn. 10: 341-356.

Janson, C.H. \& Byrne, R. (2007). What wild primates know about resources: opening up the black box. - Anim. Cogn. 10: 357-367.

Kanngiesser, P. \& Call, J. (2010). Bonobos, chimpanzees, gorillas, and orangutans use feature and spatial cues in two spatial memory tasks. - Anim. Cogn. 13: 419-430.

Kano, T. (1992). The last ape: pygmy chimpanzee behavior and ecology. — Stanford University Press, Stanford, CA.

Learmonth, A.E., Newcombe, N.S., Sheridan, N. \& Jones, M. (2008). Why size counts: children's spatial reorientation in large and small enclosures. - Dev. Sci. 11: 414-426.

Lee, S.A. \& Spelke, E. (2010). Two systems of spatial representation underlying navigation. — Exp. Brain Res. 206: 179-188.

Levinson, S.C. (1996). Frames of reference and Molyneux's question: cross-linguistic evidence. - In: Language and space. Language, speech, and communication (Bloom, P., Peterson, M.A., Nadel, L. \& Garrett, M.F., eds). MIT Press, Cambridge, MA, p. 385-436.

Levinson, S.C., Kita, S., Haun, D.B.M. \& Rasch, B.H. (2002). Returning the tables: language affects spatial reasoning. - Cognition 84: 144-188.

Li, P. \& Gleitman, L. (2002). Turning the tables: language and spatial reasoning. — Cognition 83: 265-294.

Maguire, E.A., Burgess, N., Donnett, J.G., Frackowiak, R.S.J., Frith, C.D. \& O'Keefe, J. (1998). Knowning where and getting there: a human navigation network. - Science 280: 921-923.

Majid, A., Bowerman, M., Kita, S., Haun, D. \& Levinson, S. (2004). Can language restructure cognition? The case for space. - Trends Cogn. Sci. 8: 108-114.

Malenky, R.K. \& Wrangham, R.W. (1993). A quantitative comparison of terrestrial herbaceous food consumption by Pan paniscus in the Lomako Forest, Zaire, and Pan troglodytes in the Kibale Forest, Uganda. - Am. J. Primatol. 32: 1-12.

Martin-Ordas, G., Haun, D., Colmenares, F. \& Call, J. (2010). Keeping track of time: evidence for episodic-like memory in great apes. - Anim. Cogn. 13: 331-340.

Matsuzawa, T. (2007). Comparative cognitive development. — Dev. Sci. 10: 97-103.

Matsuzawa, T., Tomonaga, M. \& Tanaka, M. (eds) (2006). Cognitive development in chimpanzees. - Springer, Tokyo. 
Mendes, N. (2008). Spatial memory in chimpanzees: single-trial learning and 24 hour and 3 month long-term memory. — Folia Primatol. 79: 283-304.

Menzel, C.R., Savage-Rumbaugh, E.S. \& Menzel, E.W. (2002). Bonobo (Pan paniscus) spatial memory and communication in a 20-hectare forest. — Int. J. Primatol. 23: 601-619.

Menzel, E.W. (1973). Chimpanzee spatial memory organization. - Science 182: 943-945.

Newcombe, N.S. \& Huttenlocher, J. (2006). Development of spatial cognition. - In: Handbook of child psychology, Vol. II: cognition, perception, and language (Damon, W., Lerner, R.M., Kuhn, D. \& Siegler, R.S., eds). Wiley, New York, NY, p. 734-776.

Newcombe, N.S. \& Ratliff, K.R. (2007). Explaining the development of spatial reorientation: modularity-plus-language versus the emergence of adaptive combination. — In: Emerging landscapes of mind: mapping the nature of change in spatial cognitive development (Plumert, J. \& Spencer, J., eds). Oxford University Press, New York, NY, p. 53-76.

Newcombe, N.S., Huttenlocher, J., Drummey, A. \& Wiley, J.G. (1998). The development of spatial location coding: place learning and dead reckoning in the second and third years. — Cogn. Dev. 13: 185-200.

Normand, E., Ban, S.D. \& Boesch, C. (2009). Forest chimpanzees (Pan troglodytes verus) remember the location of numerous fruit trees. — Anim. Cogn. 12: 797-807.

Normand, E. \& Boesch, C. (2009). Sophisticated Euclidean maps in forest chimpanzees. Anim. Behav. 77: 1195-1201.

Packard, M.G. (1996). Inactivation of hippocampus or caudate nucleus with lidocaine differentially affects expression of place and response learning. - Neurobiol. Learn. Mem. 65: 65-72.

Packard, M.G. (1999). Glutamate infused posttraining into the hippocampus or caudateputamen differentially strengthens place and response learning. — Proc. Natl. Acad. Sci. USA 96: 12881-12886.

Packard, M.G. (2009). Exhumed from thought: basal ganglia and response learning in the plus-maze. - Behav. Brain Res. 199: 24-31.

Packard, M.G. \& Goodman, J. (2013). Factors that influence the relative use of multiple memory systems. - Hippocampus 23: 1044-1052.

Pederson, E., Danziger, E., Wilkins, D.G., Levinson, S.C., Kita, S. \& Senft, G. (1998). Semantic typology and spatial conceptualization. - Language 74: 557-589.

Poldrack, R.A., Clark, M.A., Pare-Blagoev, E.J., Shohamy, D., Creso Moyan, J., Myers, C., et al. (2001). Interactive memory systems in the human brain. - Nature 414: 546.

Poldrack, R.A. \& Packard, M.G. (2003). Competition among multiple memory systems: converging evidence from animal and human brain studies. - Neuropsychologia 41: 245251.

R Development Core Team (2011). A language and environment for statistical computing. http://www.R-project.org.

Ratliff, K.R. \& Newcombe, N.S. (2008). Reorienting when clues conflct: evidence for an adaptive-combination view. - Psychol. Sci. 19: 1301-1307.

Rosati, A.G. \& Hare, B. (2012). Chimpanzees and bonobos exhibit divergent spatial memory development. — Dev. Sci. 15: 840-853. 
Rosati, A.G., Rodriguez, K. \& Hare, B. (2014). The ecology of spatial memory in four lemur species. - Anim. Cogn., in press, DOI:10.1007/s10071-014-0727-2.

Rosati, A.G., Wobber, V., Hughes, K. \& Santos, L.R. (in press). How is human cognitive development unique? - Comp. Dev. Psychol.

Sherry, D.F. \& Schacter, D.L. (1987). The evolution of multiple memory systems. — Psychol. Rev. 94: 439-454.

Shettleworth, S.J. (1998). Cognition, evolution, and behavior. - Oxford University Press, New York, NY.

Spelke, E., Lee, S.A. \& Izard, V. (2010). Beyond core knowledge: natural geometry. — Cogn. Sci. 1: 1-22.

Tolman, E.C. (1948). Cognitive maps in rats and men. - Psychol. Rev. 55: 189-208.

Wang, R.F. \& Spelke, E.S. (2002). Human spatial representation: insights from animals. Trends Cogn. Sci. 6: 376-382.

White, F.J. (1998). Seasonality and socioecology: the importance of variation in fruit abundance to bonobo sociality. — Int. J. Primatol. 19: 1013-1027.

White, F.J. \& Wrangham, R.W. (1988). Feeding competition and patch size in the chimpanzee species Pan paniscus and Pan troglodytes. — Behaviour 105: 148-163.

White, N.M. \& McDonald, R.J. (2002). Multiple parallel memory systems in the brain of the rat. - Neurobiol. Learn. Mem. 77: 125-184.

Wobber, V. \& Hare, B. (2011). Psychological health of orphan bonobos and chimpanzees in African sanctuaries. - PLoS One 6: e17147.

Wobber, V., Wrangham, R. \& Hare, B. (2010). Bonobos exhibit delayed development of social behavior and cognition relative to chimpanzees. - Curr. Biol. 20: 226-230.

Wobber, V., Herrmann, E., Hare, B., Wrangham, R. \& Tomasello, M. (2013). Differences in the early cognitive development of children and great apes. - Dev. Psychobiol., in press, DOI:10.1002/dev.21125. 